\title{
Living donor liver transplantation with total pancreatectomy and portal vein homograft replacement in a pediatric patient with advanced pancreatoblastoma
}

\author{
Jung-Man Namgoong ${ }^{1}$, Shin Hwang ${ }^{2}$, Seak Hee $\mathrm{Oh}^{3}$, Kyung Mo Kim³ ${ }^{3}$, Gil-Chun Park², \\ Chul-Soo Ahn ${ }^{2}$, Hyunhee Kwon ${ }^{1}$, Yu Jeong $\mathrm{Cho}^{1}$, and Yong Jae Kwon ${ }^{1}$ \\ ${ }^{1}$ Division of Pediatric Surgery, Department of Surgery, Asan Medical Center, University of Ulsan College of \\ Medicine, ' ${ }^{2}$ Division of Hepatobiliary Surgery and Liver Transplantation, Department of Surgery, \\ Asan Medical Center, University of Ulsan College of Medicine, ${ }^{3}$ Department of Pediatrics, Asan Medical Center, \\ University of Ulsan College of Medicine, Seoul, Korea

\begin{abstract}
Pancreatoblastoma is a malignant exocrine pancreatic tumor that is usually present in childhood. We herein present one case of pediatric living donor liver transplantation (LDLT) combined with spleen-preserving regional total pancreatectomy and portal vein homograft interposition in a 4-year-old boy with advanced pancreatoblastoma invading the portal and superior mesenteric veins. The size of the pancreatoblastoma was gradually reduced along systemic chemotherapy, thus we decided to perform surgery to remove it completely. A cold-stored fresh iliac vein homograft was prepared. Initially, a spleen-preserving distal pancreatectomy was performed. Thereafter, a completion regional total pancreatectomy was performed under superior mesenteric vein-vena cava bypass. A left liver graft from his mother was implanted according to the standardized procedures with portal vein interposition. This patient recovered uneventfully and is currently undergoing scheduled adjuvant chemotherapy. To our knowledge, this is the world-second case of pediatric LDLT for advanced pancreatoblastoma. Availability of fresh vein homografts is helpful to expand the indication of pediatric LDLT. (Ann Hepatobiliary Pancreat Surg 2020;24:78-84)
\end{abstract}

Key Words: Homograft; Pancreatectomy; Pediatric transplantation; Pancreatoblastoma

\section{INTRODUCTION}

Pancreatoblastoma is a malignant exocrine pancreatic tumor that is usually present in childhood. ${ }^{1-3}$ Its incidence of occurrence is very rare, but it is the most common pancreatic tumor in children. Pancreatoblastoma is an aggressive tumor and often unresectable at diagnosis. In order to achieve size reduction before surgical resection, neoadjuvant chemotherapy is usually necessary. ${ }^{1-3}$ Complete surgical resection is necessary to improve survival outcomes, but this is difficult in patients with extensive extra-pancreatic tumor extension or distant metastasis. Since there is only a few case reports on metastatic pancreatoblastoma in literature, standard treatments have not been established yet. $^{1-4}$ There was only one case report on liver transplantation for metastatic pancreatoblastoma in litera- ture. $^{5}$

We herein report one case of pediatric living donor liver transplantation (LDLT) combined with spleen-preserving regional total pancreatectomy and portal vein homograft interposition in a 4-year-old boy with advanced pancreatoblastoma.

\section{CASE}

The patient was a 4-year-old boy, weighing $16 \mathrm{kgs}$ and suffering from huge biopsy-proven pancreatoblastoma, which was diagnosed 9 months before (Fig. 1A). This pancreatoblastoma was partially responsive to systemic chemotherapy (Fig. 1B, C). The residual tumor occupied the pancreas and infiltrated along the main portal vein (PV) with cavernous transformation (Fig. 1D), by which

Received: January 30, 2020; Revised: February 3, 2020; Accepted: February 7, 2020

Corresponding author: Shin Hwang

Department of Surgery, Asan Medical Center, University of Ulsan College of Medicine, 88 Olympic-ro 43-gil, Songpa-gu, Seoul 05505, Korea Tel: +82-2-3010-3930, Fax: +82-2-3010-6701, E-mail: shwang@amc.seoul.kr

Copyright (C) 2020 by The Korean Association of Hepato-Biliary-Pancreatic Surgery

This is an Open Access article distributed under the terms of the Creative Commons Attribution Non-Commercial License (http://creativecommons.org/ censes/by-nc/4.0) which permits unrestricted non-commercial use, distribution, and reproduction in any medium, provided the original work is properly cited. Annals of Hepato-Biliary-Pancreatic Surgery - pISSN: 2508-5778 - elSSN: 2508-5859 
the liver could not be saved during pancreatectomy because PV reconstruction was not possible. Complete tumor removal and subsequent adjuvant chemotherapy appeared to be the only therapeutic option to achieve prolonged survival. Therefore, we decided to perform LDLT in combination with regional total pancreatectomy. Since an iliac vein homograft was necessary to replace the PV and superior mesenteric vein (SMV), we had to wait until a cold-stored fresh iliac vein homograft was available. After waiting for three weeks, we obtained an iliac vein homograft that was recovered from a deceased organ donor. Thereafter, we performed LDLT operation using a left liver graft from his 40-year-old mother.

After laparotomy, the whole abdomen was examined to identify any missed peritoneal seeding and we confirmed absence of metastasis (Fig. 2). Initially, the body of the pancreas was meticulously dissected and we performed spleen-preserving distal pancreatectomy with extensive dissection of the celiac axis. Thereafter, the duodenum, pancreas head and uncinated process were meticulously dissected to isolate the SMV (Fig. 3). Since the PV was completely occluded and PV collaterals should be trans-
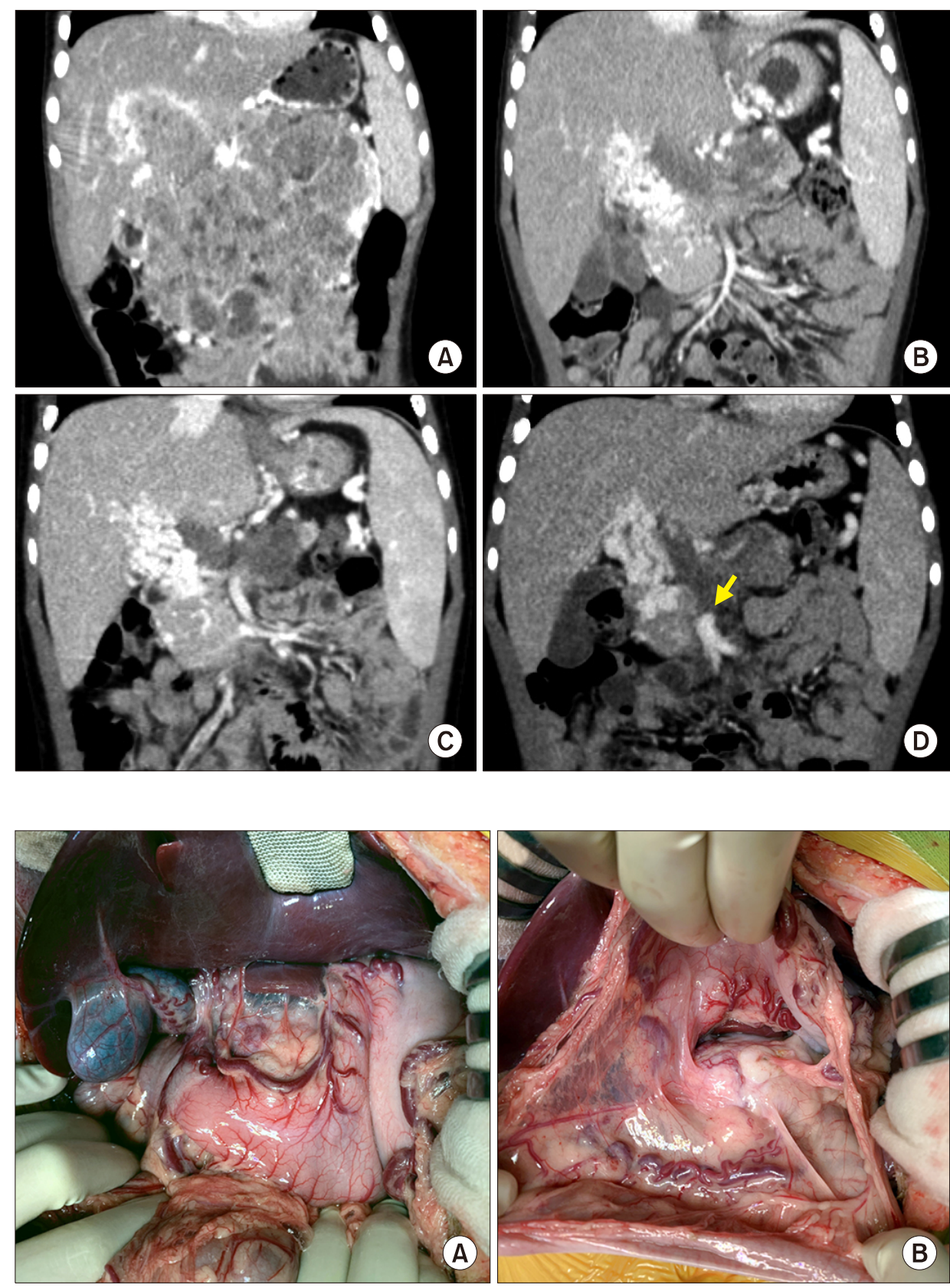

Fig. 1. Pretransplant computed tomography findings. The size of pancreatoblastoma was gradually reduced according to the neoadjuvant chemotherapy, showing serial size changes as follows: at diagnosis (A), five months before transplantation, (B) one month before transplantation, and (C) one week before transplantation (D). An arrow indicates the proximal end of the superior mesenteric vein.

Fig. 2. Intraoperative photographs of the recipient's abdomen. No peritoneal seeding was identified at the mesenteric side (A) and around the pancreas (B). 


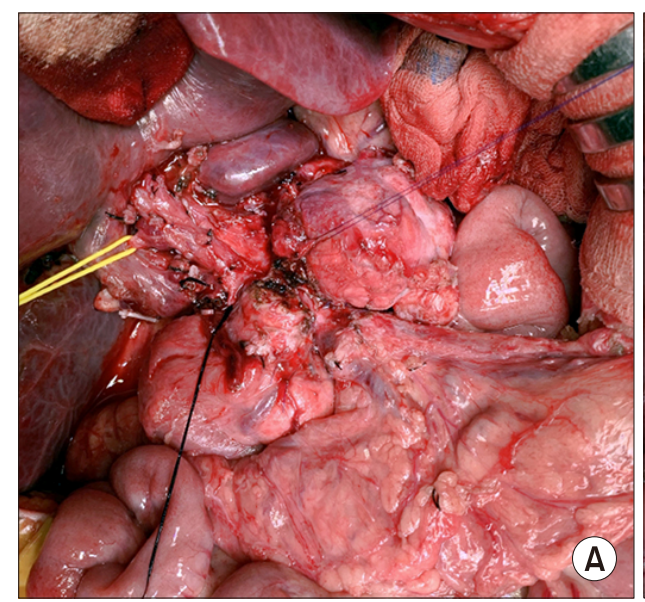

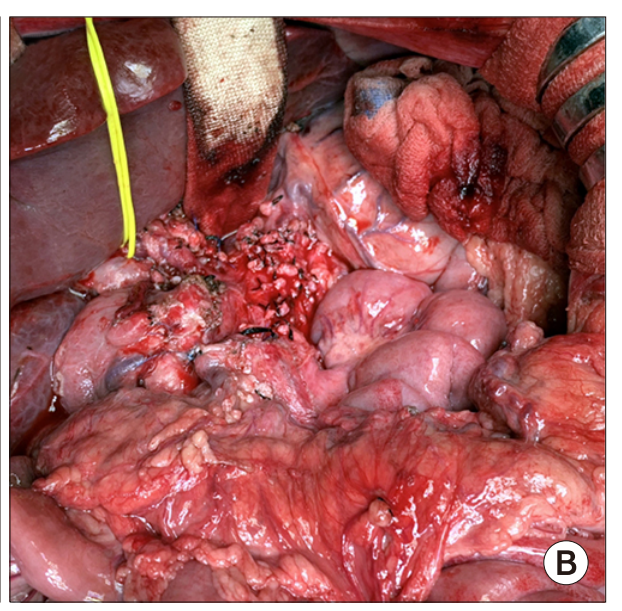

Fig. 3. Intraoperative photographs of spleen-preserving distal pancreatectomy. The pancreas body was transected to facilitate pancreatic resection (A). The celiac axis area was completely dissected (B).
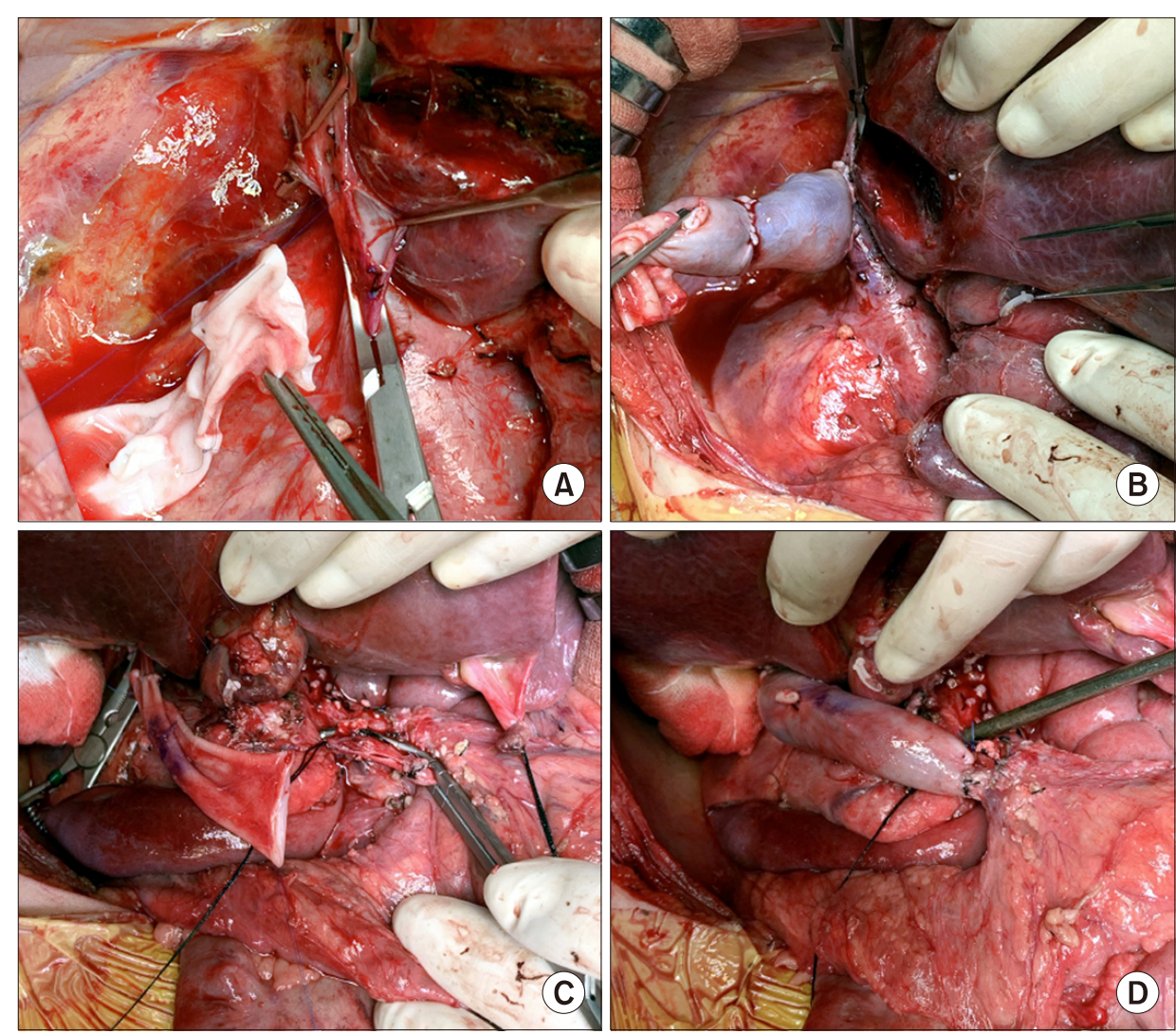

Fig. 4. Intraoperative photographs of the superior mesenteric vein-vena cava bypass using a cold-stored fresh iliac vein homograft. The vein graft is anastomosed to the retrohepatic inferior vena cava (A and B). The vein conduit was anastomosed to the superior mesenteric vein as an end-to-side fashion (C and D). ected to perform regional total pancreatectomy, the prepared iliac vein homograft was anastomosed to the retrohepatic inferior vena cava as an end-to-side fashion (Fig. 4A, B), which would be used for SMV-vena cava bypass. The main PV and proximal portion of the SMV was completely occluded, thus the uppermost portion of the intact SMV was incised and the iliac vein conduit was anastomosed as an end-to-side fashion for SMV-caval bypass (Fig. 4C, D). The transected SMV stump margin was sent for frozen-section biopsy, which was reported to be tu- mor-negative. Under the SMV-caval bypass, regional total pancreatectomy was meticulously performed with preservation of the common hepatic artery. Repeated frozen-section biopsies were performed during peripancreatic dissection to ensure that no tumor mass was left behind. Eight hours were taken for completion of the spleen-preserving regional total pancreatectomy and SMV-caval bypass (Fig. 5).

Thereafter, a 415-weighing extended left liver graft was harvested. The graft hepatic vein orifices were unified 
with an iliac vein patch (Fig. 6A). The recipient's native liver was removed with clamping of the SMV-caval bypass. The hepatic vein orifice of the left liver graft was anastomosed to the unified orifice of the three recipient hepatic veins (Fig. 6B). The iliac vein conduit from the SMV was transected and anastomosed to the graft portal vein according to the standardized techniques (Fig. 6C, D). Reconstruction of the single left hepatic artery and Roux-en-Y hepaticojejunostomy were sequentially performed (Fig. 7). The pathology report presented that there was residual viable pancreatoblastoma at the pancreas tis- sue without lymph node metastasis (Fig. 8).

This patient recovered uneventfully (Fig. 9), and is doing well for three months to date. He is currently undergoing scheduled adjuvant chemotherapy. Type I diabetes mellitus and pancreatic exocrine insufficiency are well controlled by regular administration of insulin and pancreatic enzyme supplement.

\section{DISCUSSION}

Pancreatoblastoma is a rare primary malignant tumor
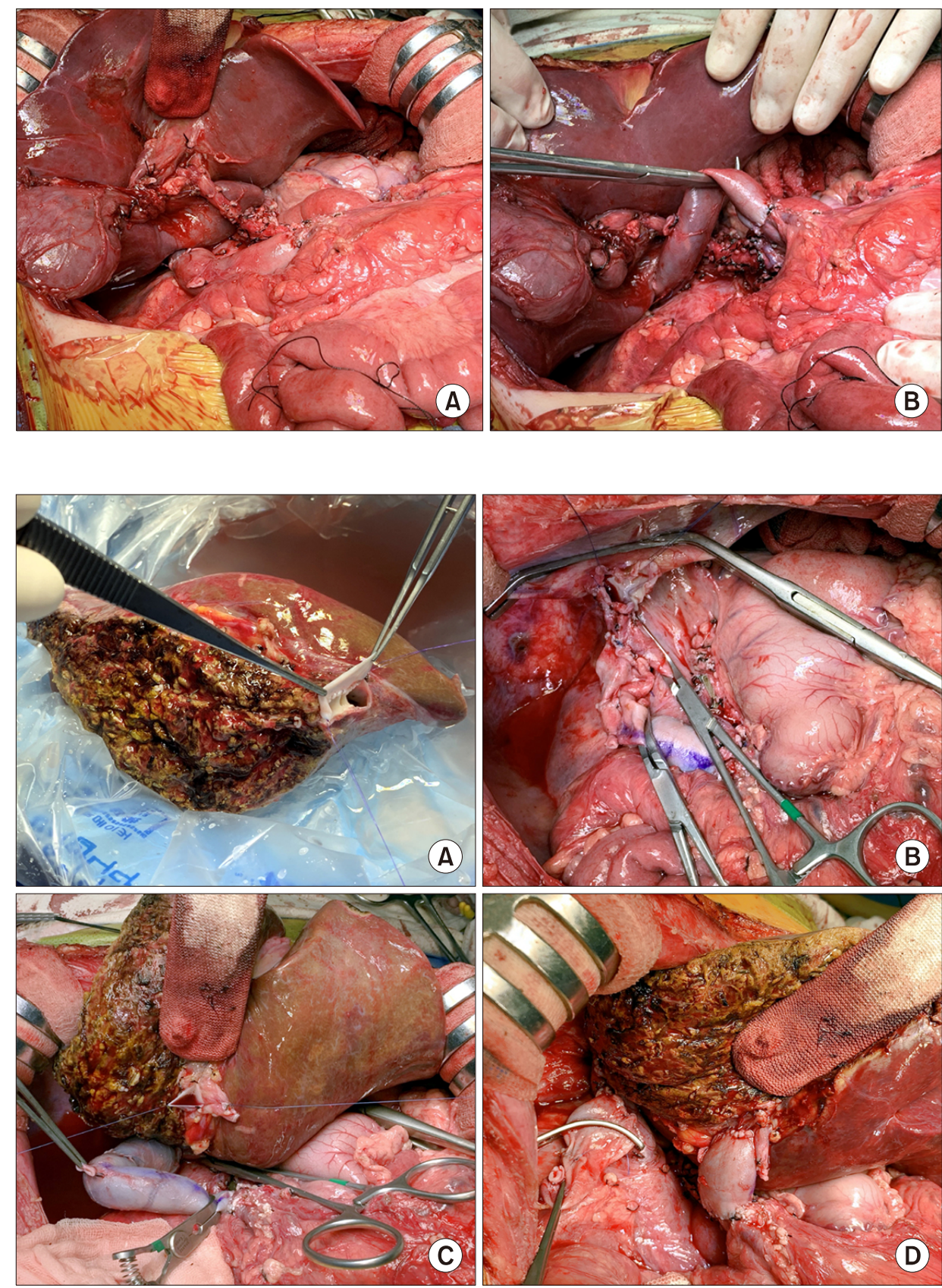

Fig. 5. Intraoperative photographs of spleen-preserving regional total pancreatectomy. The common hepatic artery was preserved with extensive lymph node dissection (A). Superior mesenteric vein-caval bypass was maintained during pancreatectomy (B).

Fig. 6. Intraoperative photographs of the left liver graft implantation. The graft hepatic vein orifices were unified with an iliac vein patch (A). The recipient's native liver was removed with clamping of the superior mesenteric vein-caval bypass (B). The venous bypass was clamped (C). The iliac vein conduit was anastomosed to the gra$\mathrm{ft}$ portal vein (D). 


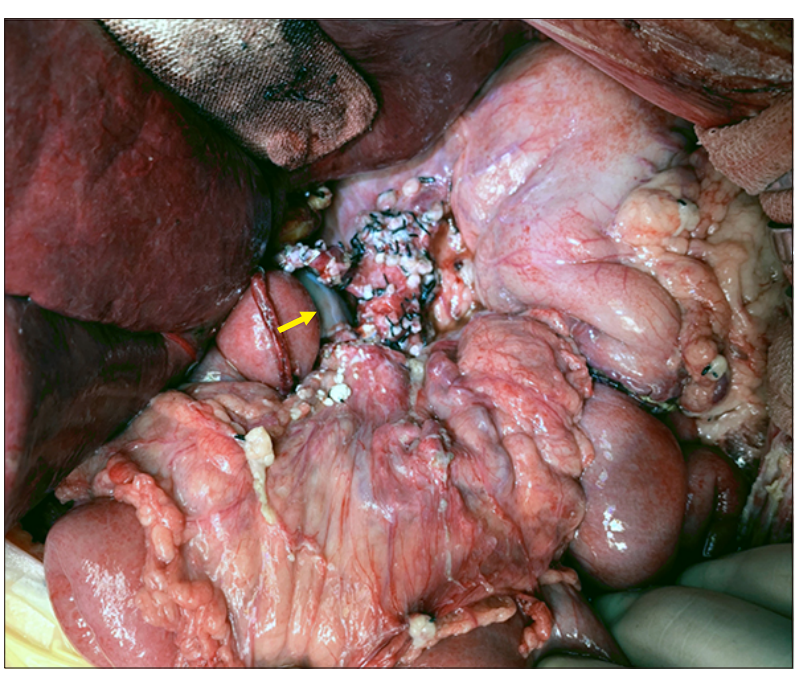

Fig. 7. Intraoperative photographs of the recipient's abdomen after graft implantation. The extent of dissection was visualized. An arrow indicates the interposed iliac vein homograft.

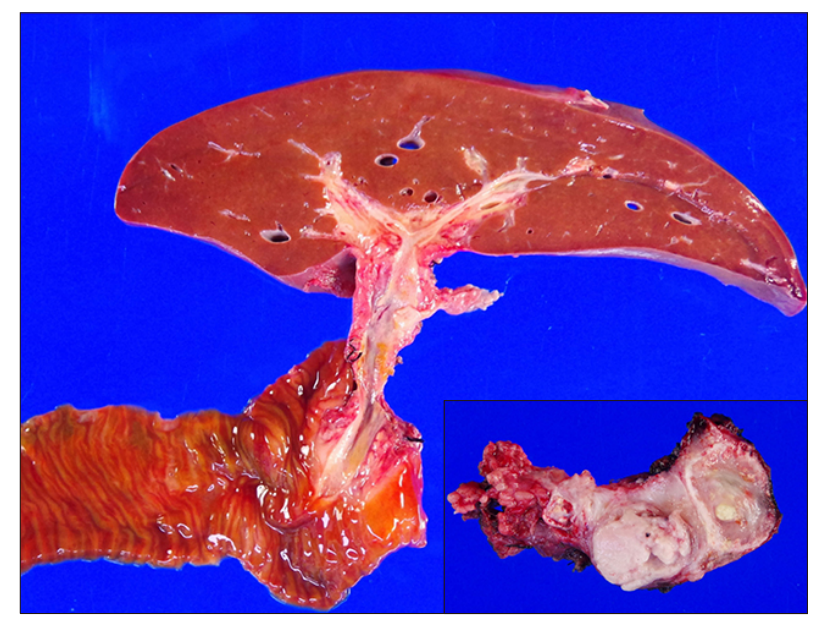

Fig. 8. Gross photographs of the resected specimens including the liver, pancreas and duodenum.

that occurs during childhood with a peak incidence around 5 years of age. Its annual incidence was 0.004 new cases per 100,000 population, but it is the most common pancreatic tumor in children. ${ }^{1-5}$ Complete surgical resection is necessary to improve the survival outcomes. The overall 5-year patient survival rates were reported to be $30-50 \%{ }^{4}$ However, the prognosis of patients with advanced pancreatoblastoma with or without metastasis was poor. About $30 \%$ of patients present with metastasis and few of them become long-term survivors. One study with 17 cases with metastatic pancreatoblastoma reported $25 \%$ of 5-year survival rate.

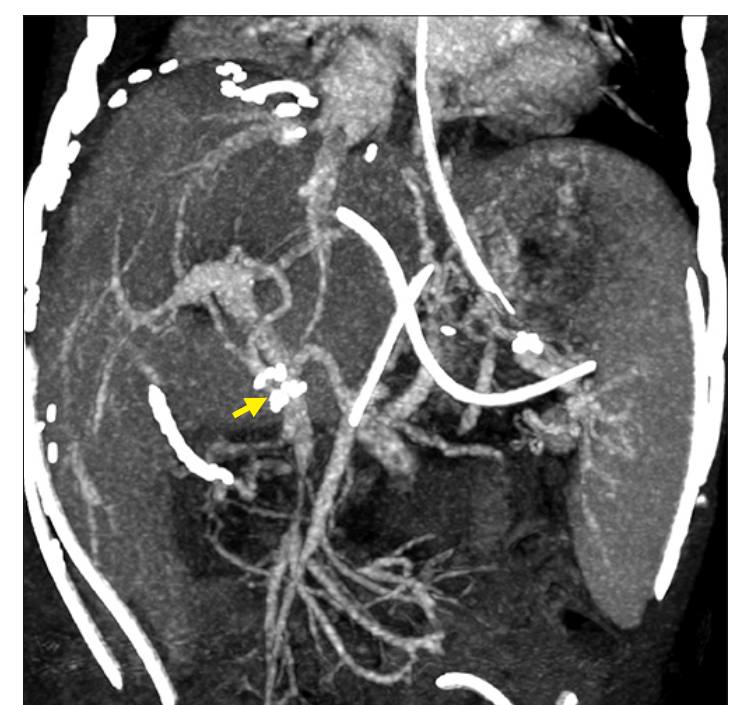

Fig. 9. Posttransplant computed tomography finding showing streamlined reconstruction figures of the graft hepatic and portal veins taken one week after transplantation. An arrow indicates the interposed iliac vein homograft.

The treatment outcomes of the pancreatoblastoma did not correlate with the tumor site and size, but was strongly influenced by the feasibility of complete resection of the tumor. ${ }^{4}$ To achieve complete tumor removal in advanced pancreatoblastoma with or without metastasis, LT can be combined with pancreatectomy. There is only one case of LT for advanced pancreatoblastoma in literature. ${ }^{5}$ The 4-year-old boy had pancreatoblastoma with liver metastasis. After downsizing with neoadjuvant chemotherapy, the patient underwent staged operations. The first operation was a subtotal pancreatectomy to remove the pancreatic mass. Two days later, the resection margins were pathologically proven to be tumor-negative and vascular structures were not involved in the pathology report. Thereafter, LDLT operation was performed with a left lateral section graft from his aunt. He underwent postoperative adjuvant chemotherapy and is alive and healthy, and has now been tumor-free for 7 years with no tumor relapse. ${ }^{5}$

To our knowledge, our case is the second case of LT for advanced pancreatoblastoma. Unlike the above mentioned world-first case, ${ }^{5}$ the primary reason of performing LT in our patient was because we could not save the liver due to hilar invasion with complete PV occlusion and collateral formation. After extensive discussion, we finally decided to perform LDLT and regional total pan- 
createctomy concurrently. Two-staged operations were performed to decide whether the first subtotal pancreatectomy was curative in the case mentioned above. In contrast, we performed one-stage operation with frequent intraoperative frozen-section biopsies. According to our large experience on surgery for hepatobiliary and pancreatic malignancies, ${ }^{6}$ we progressed the initial distal pancreatectomy very slowly because most of the pancreatic and peripancreatic tissues were heavily adhered and vessels were fragile. If the regional lymph nodes and neural plexus at the celiac axis had been tumor-positive, we would have cancelled the LDLT operation because such $\mathrm{R} 1 / \mathrm{R} 2$ resection would definitely increase the risk of post-transplant tumor recurrence. ${ }^{7,8}$ After confirming that the initial spleen-preserving distal pancreatectomy was microscopically curative (R0 resection), we started additional procedures for regional total pancreatectomy and LDLT.

In our case, we used the SMV-caval bypass to securely perform pancreatoduodenectomy. Since the main PV was completely occluded and abundant collaterals were formed, secure peripancreatic dissection was difficult due to fragile bleeding. The SMV-caval bypass was effective in preventing dissection-associated bleeding, which enabled us to perform meticulous removal of the tumor. If a lengthy iliac vein homograft was not available, such bypass would have not been possible because any prosthetic vascular graft cannot be anastomosed to the thin-walled SMV stump and such grafts cannot be used for PV interposition in a pediatric patient.

After deciding to perform LDLT with SMV-caval bypass in this case, we had to wait three weeks to obtain a fresh cold-stored iliac vein homograft, which was recovered from a deceased organ donor. The homograft was retrieved after obtaining a written consent on tissue donation and registered it to the tissue bank at our institution. At this time, we urgently scheduled the LDLT operation just after confirmation of negative bacteria culture finding. To use the iliac vein homograft in a fresh state, it can be stored in $4{ }^{\circ} \mathrm{C}$ within 7 days instead of cryopreservation. In pediatric recipients, various vein homografts, such as iliac vein, inferior vena cava and other large-sized veins, can be used depending on the body size of the recipient and availability of vessel graft. We suggest that the use of cryopreserved veins should be avoided if possible for pediatric patients because they can degenerate over time.' Availability of fresh vein homografts is helpful to expand the indication of pediatric LDLT, especially for young patients with advanced malignancies.

\section{ORCID}

Jung-Man Namgoong: https://orcid.org/0000-0002-92377440

Shin Hwang: https://orcid.org/0000-0002-9045-2531

Seak Hee Oh: https://orcid.org/0000-0002-9672-8877

Kyung Mo Kim: https://orcid.org/0000-0001-7896-6751

Gil-Chun Park: https://orcid.org/0000-0003-1631-3258

Chul-Soo Ahn: https://orcid.org/0000-0002-3844-3646

Hyunhee Kwon: https://orcid.org/0000-0001-6647-9155

Yu Jeong Cho: https://orcid.org/0000-0001-6823-2746

Yong Jae Kwon: https://orcid.org/0000-0001-9490-1229

\section{AUTHOR CONTRIBUTIONS}

Conceptualization: SH, JMN.

Data curation: SHO, KMK.

Formal analysis: GCP, CSA, HK, YJC, YJK.

Funding acquisition: Not applicable.

Methodology: JMN, SH.

Project administration: SH.

Visualization: JMN, SH.

Writing - original draft: JMN, SH.

Writing - review \& editing: SH.

\section{REFERENCES}

1. Dhebri AR, Connor S, Campbell F, Ghaneh P, Sutton R, Neoptolemos JP. Diagnosis, treatment and outcome of pancreatoblastoma. Pancreatology 2004;4:441-451; discussion 452-453.

2. Défachelles AS, Martin De Lassalle E, Boutard P, Nelken B, Schneider P, Patte C. Pancreatoblastoma in childhood: clinical course and therapeutic management of seven patients. Med Pediatr Oncol 2001;37:47-52.

3. Glick RD, Pashankar FD, Pappo A, Laquaglia MP. Management of pancreatoblastoma in children and young adults. J Pediatr Hematol Oncol 2012;34 Suppl 2:S47-S50.

4. Bien E, Godzinski J, Dall'igna P, Defachelles AS, StachowiczStencel T, Orbach D, et al. Pancreatoblastoma: a report from the European cooperative study group for paediatric rare tumours (EXPeRT). Eur J Cancer 2011;47:2347-2352.

5. Ghaffarian AA, Book L, Meyers RL. Liver transplant for metastatic pancreatoblastoma: 7-year event-free survival after chemotherapy, pancreatectomy, complete hepatectomy, and liver transplant. Pediatr Transplant 2018. doi: 10.1111/petr.13098. 
6. Cho HD, Hwang S, Lee YJ, Park KM, Kim KH, Kim JC, et al. Changes in the types of liver diseases requiring hepatic resection: a single-institution experience of 9016 cases over a 10-year period. Korean J Hepatobiliary Pancreat Surg 2016;20: 49-52.

7. Ibuka S, Uehara S, Ueno T, Oue T, Miyamura T, Hashii Y, et al. Complete resection of pancreatoblastoma with portal vein obstruction after high-dose chemotherapy: a case report. J Pediatr Hematol Oncol 2017;39:e275-e278.
8. Lindholm EB, Alkattan AK, Abramson SJ, Price AP, Heaton TE Balachandran VP, et al. Pancreaticoduodenectomy for pediatric and adolescent pancreatic malignancy: a single-center retrospective analysis. J Pediatr Surg 2017;52:299-303.

9. Sugawara Y, Makuuchi M, Tamura S, Matsui Y, Kaneko J, Hasegawa $\mathrm{K}$, et al. Portal vein reconstruction in adult living donor liver transplantation using cryopreserved vein grafts. Liver Transpl 2006;12:1233-1236. 